\title{
The joint distribution of the sample minimum and maximum from a smooth distribution on w1,w2
}

DOI:

10.1016/j.jkss.2018.02.003

\section{Document Version}

Accepted author manuscript

Link to publication record in Manchester Research Explorer

\section{Citation for published version (APA):}

Withers, C. S., \& Nadarajah, S. (2018). The joint distribution of the sample minimum and maximum from a smooth distribution on w1,w2. Journal of the Korean Statistical Society. https://doi.org/10.1016/j.jkss.2018.02.003

\section{Published in:}

Journal of the Korean Statistical Society

\section{Citing this paper}

Please note that where the full-text provided on Manchester Research Explorer is the Author Accepted Manuscript or Proof version this may differ from the final Published version. If citing, it is advised that you check and use the publisher's definitive version.

\section{General rights}

Copyright and moral rights for the publications made accessible in the Research Explorer are retained by the authors and/or other copyright owners and it is a condition of accessing publications that users recognise and abide by the legal requirements associated with these rights.

\section{Takedown policy}

If you believe that this document breaches copyright please refer to the University of Manchester's Takedown Procedures [http://man.ac.uk/04Y6Bo] or contact uml.scholarlycommunications@manchester.ac.uk providing relevant details, so we can investigate your claim.

\section{OPEN ACCESS}




\title{
The joint distribution of the sample minimum and maximum from a smooth distribution on $\left[w_{1}, w_{2}\right]$
}

by

\author{
Christopher S. Withers, Industrial Research Limited, Lower Hutt, New Zealand \\ email: kit.withers@gmail.com \\ Saralees Nadarajah, University of Manchester, Manchester M13 9PL, UK \\ email: mbbsssn2@manchester.ac.uk
}

\begin{abstract}
We give expansions in inverse powers of the sample size $n$ for the joint distribution function of the sample extremes when sampling from any smooth distribution on a finite interval when its density function is positive at its end points.
\end{abstract}

Keywords: Density function; Maximum; Moments

\section{Introduction}

Many variables in real life take values in finite intervals. The best known examples are proportions. Often interest is on extreme values of such variables, for example, lowest and highest proportions of people affected by a deadly disease.

Suppose $X_{1}, X_{2}, \ldots, X_{n}$ is a random sample from a distribution on a finite interval. Suppose $Z_{n 1}$ denotes a suitably normalized $\min \left(X_{1}, X_{2}, \ldots, X_{n}\right)$ and belongs to the Weibull domain of attraction such that

$$
\operatorname{Prob}\left(Z_{n 1}>z_{1}\right) \rightarrow e^{-z_{1}}
$$

as $n \rightarrow \infty$. Suppose $Z_{n 2}$ denotes a suitably normalized $-\max \left(X_{1}, X_{2}, \ldots, X_{n}\right)$ and belongs to the Weibull domain of attraction such that

$$
\operatorname{Prob}\left(Z_{n 2}>z_{2}\right) \rightarrow e^{-z_{2}}
$$

as $n \rightarrow \infty$. Both (1) and (2) hold if for example the sample is from the uniform [0,1] distribution.

There is much work on the asymptotic joint distribution of sample minimum and sample maximum. For example, Leadbetter et al. (1983) showed under certain general conditions that sample

minimum and sample maximum are asymptotically independent. If (1) and (2) hold then under certain general conditions

$$
\begin{gathered}
\operatorname{Prob.}\left(Z_{n 1}>z_{1}, Z_{n 2}>z_{2}\right) \rightarrow e^{-z_{1}-z_{2}} \\
\frac{\partial}{\partial z_{1}} \frac{\partial}{\partial z_{2}} \operatorname{Prob.}\left(Z_{n 1} \leq z_{1}, Z_{n 2} \leq z_{2}\right) \rightarrow e^{-z_{1}-z_{2}}
\end{gathered}
$$

and

$$
E Z_{n 1}^{r} Z_{n 2}^{s} \rightarrow r ! s !
$$


as $n \rightarrow \infty$. In practice, $n$ is finite. It can never be infinity. So, models based on (3)-(5) cannot be realistic. Realistic models can be obtained by deriving expansions for the joint distribution of the minimum and maximum. Liao and Peng (2015) and Lu and Peng (2017) give the most recent work on the asymptotics of the joint distribution of the minimum and maximum.

The aim of this note is to derive expansions for the distributions of the minimum and maximum of a random sample of values in a finite interval. We derive expansions for the joint distribution function, joint density function and product moments. These expansions can be used for improved statistical modeling of the minimum and maximum. The expansions for the joint distribution function can be used for improved percentile estimation. For instance, by Corollary 3.1 below,

$$
\operatorname{Prob} .\left(Z_{n 1}>z_{1}, Z_{n 2}>z_{2}\right)=e^{-z_{1}-z_{2}}\left(1+Q_{1} n^{-1}\right)
$$

which can be expected to perform better than (1). The expansions for the joint density function can be used for improved maximum likelihood estimation. For instance, by Corollary 3.1 below,

$$
\frac{\partial}{\partial z_{1}} \frac{\partial}{\partial z_{2}} \operatorname{Prob} .\left(Z_{n 1} \leq z_{1}, Z_{n 2} \leq z_{2}\right)=e^{-z_{1}-z_{2}}\left(1+q_{1}^{\prime} n^{-1}\right)
$$

which can be expected to perform better than (2). The expansions for the moments can be used for improved moments estimation. For instance, by Corollary 3.1 below,

$$
E Z_{n 1}^{r} Z_{n 2}^{s}=r ! s !+M_{r s 1} n^{-1}
$$

which can be expected to perform better than (3).

The known work giving expansions for the joint distribution of sample minimum and sample maximum has focused on specific distributions or processes: a Brownian motion (Choi and Roh, 2013); the normal distribution (Shiryaeva, 2013); autoregressive process of order one (Withers and Nadarajah, 2015); and so on. We are aware of no work giving expansions for the joint distribution for general classes.

Let $X_{1}, \ldots, X_{n}$ be a random sample with minimum $m_{n}$ and maximum $M_{n}$ from any distribution function $F(x)$ on a finite interval $\left[w_{1}, w_{2}\right]$ with finite derivatives $\left\{F_{. r}(x), r \geq 0\right\}$ at its endpoints $w_{1}, w_{2}$. However, $F_{. r}(x)$ need not exist in $\left(w_{1}, w_{2}\right)$.

Section 2 gives the joint distribution function and product moments of

$$
\mathbf{Y}_{n}=\left(Y_{n 1}, Y_{n 2}\right)=n\left(m_{n}-w_{1}, w_{2}-M_{n}\right)
$$

in terms of

$$
A_{r}=F_{. r}\left(w_{1}\right) / r !, B_{r}=F_{. r}\left(w_{2}\right) / r !
$$

as expansions in powers of $n^{-1}$. Note that

$$
Y_{n 1} \geq 0, Y_{n 2} \geq 0, Y_{n 1}+Y_{n 2} \leq e_{n}=n\left(w_{2}-w_{1}\right),
$$

and $B_{0}=1, A_{1} \geq 0, B_{1} \geq 0$. We assume that $A_{0}=0$ (that is $F$ does not have an atom at $w_{1}$ ), and $A_{1}>0, B_{1}>0$. So, the density function of $F(x)$ is positive at its end points. For example, this rules out the beta distribution except for the uniform distribution.

Section 3 gives the joint distribution function and product moments of

$$
\mathbf{Z}_{n}=\left(Z_{n 1}, Z_{n 2}\right)=\left(A_{1} Y_{n 1}, B_{1} Y_{n 2}\right)=n\left(A_{1}\left(m_{n}-w_{1}\right), B_{1}\left(w_{2}-M_{n}\right)\right)
$$


in terms of

$$
a_{r}=\bar{a}_{r}=A_{r} / A_{1}^{r}, b_{r}=B_{r} / B_{1}^{r}, \bar{b}_{r}=(-1)^{r} b_{r}
$$

as expansions in powers of $n^{-1}$ under the same conditions. This considerably simplifies the results of Section 2. Sections 4 and 5 give the distribution function and moments of the marginals of $\mathbf{Y}_{n}$ and $\mathbf{Z}_{n}$ under the same conditions. Section 6 shows that $\operatorname{corr}\left(Z_{n 1}, Z_{n 2}\right)=O\left(n^{-1}\right)$ and outlines how to deal with the case $A_{1}=0$ or $B_{1}=0$.

We need some notations. For $n, r=0,1, \ldots$, set $(n)_{r}=n ! /(n-r) !=n(n-1) \cdots(n-r+1)$. Let $\delta_{i, j}=I(i=j)$ denote the Kronecker delta function. For a sequence $\mathbf{c}=\left(c_{1}, c_{2}, \ldots\right)$ of real or complex numbers, define the partial ordinary Bell polynomial, $\widetilde{B}_{r, k}=\widetilde{B}_{r, k}(\mathbf{c})$, by

$$
\left(\sum_{r=1}^{\infty} c_{r} t^{r}\right)^{k}=\sum_{r=k}^{\infty} \widetilde{B}_{r, k} t^{r}
$$

for $t \in C$, the plane of complex numbers, and $k=0,1, \ldots$ Comtet (1974) tabled $\widetilde{B}_{r, k}$ on page 309 for $1 \leq r \leq 10$. Bell polynomials are valuable tools for handling power series.

\section{The joint distribution and moments of $\mathbf{Y}_{n}$}

Here, we assume that (7) holds with $A_{1}>0, B_{1}>0$. For $w_{1} \leq x \leq y \leq w_{2}$, the joint distribution function of $\left(m_{n}, M_{n}\right)$ is

$$
P_{n}=\operatorname{Prob} .\left(x<m_{n}, M_{n} \leq y\right)=\Delta^{n}, \quad \operatorname{Prob} .\left(m_{n} \leq x, M_{n} \leq y\right)=F(y)^{n}-\Delta^{n}
$$

with marginals

$$
P_{n 1}=\operatorname{Prob} .\left(m_{n} \leq x\right)=1-[1-F(x)]^{n}, P_{n 2}=\operatorname{Prob} .\left(M_{n} \leq y\right)=F(y)^{n},
$$

where $\Delta=F(y)-F(x)$. Now transform $(x, y)$ to $(a, b)$, where $a=n\left(x-w_{1}\right), b=n\left(w_{2}-y\right)$, that is, $x=w_{1}+a / n, y=w_{2}-b / n$. So, $a \geq 0, b \geq 0, a+b \leq e_{n}$ and $\mathbf{Y}_{n}$ of (6) has joint distribution function

$$
P_{n}=\operatorname{Prob} .\left(Y_{n 1}>a, Y_{n 2} \geq b\right)=\Delta^{n}
$$

with marginals

$$
P_{n 1}=\operatorname{Prob.}\left(Y_{n 1}>a\right), P_{n 2}=\operatorname{Prob.}\left(Y_{n 2} \geq b\right) .
$$

In terms of the end derivatives of (7), we have the Taylor expansions

$$
\begin{aligned}
& F(x)=\sum_{r=1}^{\infty}(a / n)^{r} A_{r}, F(y)=1+\sum_{r=1}^{\infty}(-b / n)^{r} B_{r}, \Delta=1-S, \\
& S=1-F(y)+F(x)=\sum_{r=1}^{\infty} c_{r} n^{-r}, c_{r}=a^{r} A_{r}-(-b)^{r} B_{r} .
\end{aligned}
$$

To regain symmetry, set

$$
\bar{A}_{r}=A_{r}, \bar{B}_{r}=(-1)^{r} B_{r}
$$

so that

$$
c_{r}=a^{r} \bar{A}_{r}+b^{r} \bar{B}_{r} .
$$




\section{Lemma 2.1 If}

$$
S=\sum_{r=1}^{\infty} c_{r} n^{-r}
$$

then

$$
n \ln (1-S)=-n \sum_{r=1}^{\infty} C_{r} n^{-r}
$$

for

$$
C_{r}=C_{r}(\mathbf{c})=\sum_{k=1}^{r} \widetilde{B}_{r, k}(\mathbf{c}) / k
$$

and $\mathbf{c}=\left(c_{1}, c_{2}, \ldots\right)$. Furthermore, if

$$
T=\sum_{i=1}^{\infty} d_{i} n^{-i}
$$

then

$$
\exp (-T)=\sum_{i=0}^{\infty} Q_{i} n^{-i}
$$

for

$$
Q_{i}=\sum_{k=0}^{i}(-1)^{k} \widetilde{B}_{i, k}(\mathbf{d}) / k !
$$

and $\mathbf{d}=\left(d_{1}, d_{2}, \ldots\right)$.

Theorem 2.1 gives expansions for the joint distribution function of $\mathbf{Y}_{n}$. and the joint density function of $\mathbf{Y}_{n}$. An expansion for the product moments of $\mathbf{Y}_{n}$ is given in Theorem 2.2.

In Theorem 2.1, $c_{r}, C_{r}, d_{r-1}$ are polynomials of degree $r$ in $(a, b) . Q_{i}$ is a polynomial of degree $2 i$ in $(a, b)$. For $f$ a function of $(a, b)$, set

$$
\partial_{a}=\partial / \partial a, f_{. a}=\partial_{a} f, f_{. a b}=\partial_{a} \partial_{b} f, J_{1}=\partial_{a}-A_{1}, J_{2}=\partial_{b}-B_{1} .
$$

If $J=J_{1} J_{2}$ then

$$
J f=f_{. a b}-f_{. b} A_{1}-f_{. a} B_{1}+A_{1} B_{1} f
$$

and

$$
J f g=f_{. a b} g+f_{. a} g_{. b}+g_{. a b} f+g_{. a} f_{. b}-B_{1}\left(f_{. a} g+f g_{. a}\right)-A_{1}\left(f_{. b} g+f g_{. b}\right)+A_{1} B_{1} f g .
$$

So,

$$
J a^{i}=-i a^{i-1} B_{1}+a^{i} A_{1} B_{1}, J a^{i} b^{j}=i j a^{i-1} b^{j-1}-a^{i} j b^{j-1} A_{1}-b^{i} j a^{j-1} B_{1}+a^{i} b^{j} A_{1} B_{1} .
$$


Theorem 2.1 For $c_{r}$ of (14) and $C_{r}$ of (16), set

$$
d_{i}=C_{i+1},
$$

and $Q_{i}(a, b)=Q_{i}$ of (17). For $a \geq 0, b \geq 0$ and $a+b \leq e_{n}$, where $e_{n}$ is given by (8), $\mathbf{Y}_{n}$ has joint distribution function

$$
\operatorname{Prob.}\left(Y_{n 1}>a, Y_{n 2} \geq b\right)=e^{-c_{1}} U_{n},
$$

where

$$
U_{n}=U_{n}(a, b)=\sum_{i=0}^{\infty} Q_{i} n^{-i} .
$$

For $c_{r}$ of (14), $Q_{i}$ of (17) and $J$ of (19), $\mathbf{Y}_{n}$ of (6) has joint density function

$$
p_{n}(a, b)=\partial_{a} \partial_{b} P_{n}=e^{-c_{1}} J U_{n},
$$

where

$$
J U_{n}=\sum_{i=0}^{\infty} q_{i}(a, b) n^{-i}
$$

and $q_{i}(a, b)=J Q_{i}$.

Proof: By (11), (15) and (17), $P_{n}$ is equal to the left hand side of (22) for $S$ of (12),

$$
\ln P_{n}=n \ln (1-S)=-C_{1}-T
$$

for

$$
T=\sum_{r=2}^{\infty} C_{r} n^{1-r}=\sum_{i=1}^{\infty} d_{i} n^{-i} .
$$

So, $P_{n}=\exp \left(-C_{1}\right) \exp (-T)$ for $C_{1}=c_{1}$. Now apply the lemma. The expansion for the joint density function follows by differentiation.

Note that $q_{i}$ is a polynomial of degree $2 i$ in $(a, b)$, say

$$
q_{i}(a, b)=\sum_{j+k \leq 2 i} q_{i j k} a^{j} b^{k} .
$$

For $q=q(a, b)$ any function of $a, b$, set

$$
\begin{aligned}
& \mathcal{F} q=\int_{0}^{\infty} e^{-a A_{1}} d a \int_{0}^{\infty} e^{-b B_{1}} q(a, b) d b, \\
& \mathcal{F}_{r s} q=\mathcal{F} a^{r} b^{s} q, F_{r s}=\mathcal{F}_{r s} 1=r ! s ! A_{1}^{-r-1} B_{1}^{-s-1} .
\end{aligned}
$$

Lemma 2.2 With $A_{1}, B_{1}$ of (7) and $J$ of (19),

$$
\mathcal{F} J a^{i} b^{j}=0,
$$




$$
\mathcal{F}_{r 0} J a^{i}= \begin{cases}r(r+i-1) ! A_{1}^{-r-i}, & r+i \neq 0 \\ 1, & i=r=0\end{cases}
$$

and

$$
\mathcal{F}_{r s} J a^{i} b^{j}= \begin{cases}r s(r+i-1) !(s+j-1) ! A_{1}^{-r-i} B_{1}^{-s-j}, & (r+i)(s+j) \neq 0, \\ r(r+i-1) ! A_{1}^{-r-i}, & s=j=0, r+i \neq 0, \\ s(s+j-1) ! B_{1}^{-s-j}, & r=i=0, s+j \neq 0, \\ 1, & r=s=i=j=0 .\end{cases}
$$

The proof of the next theorem can be used to give exact expansions for the moments of $\mathbf{Y}_{n}$, see Appendix A. We now introduce the notation $a_{n} \approx b_{n}$ if and only if $a_{n}=b_{n}+O\left(e^{-n g}\right)$, where $g>0$ does not depend on $n$.

Theorem 2.2 For $p_{n}, q_{i}$ of Theorem 2.1, $q_{i j k}$ of (23) and $\mathcal{F}_{r s}, F_{r s}$ of (24)-(25), $\mathbf{Y}_{n}$ of (6) has $(r, s)$ moment

$$
E Y_{n 1}^{r} Y_{n 2}^{s} \approx \mathcal{F}_{r s} p_{n}(a, b)=\sum_{i=0}^{\infty} m_{r s i} n^{-i}
$$

for

$$
m_{r s i}=\mathcal{F}_{r s} q_{i}(a, b)=\sum_{j+k \leq 2 i} q_{i j k} F_{r+j, s+k}
$$

by (23).

Proof: For $e_{n}$ of (8),

$$
E Y_{n 1}^{r} Y_{n 2}^{s}=\int_{0}^{e_{n}} a^{r} d a \int_{0}^{e_{n}-a} e^{-c_{1}} b^{s} p_{n}(a, b) d b=\sum_{i=0}^{\infty} m_{n r s i} n^{-i}
$$

where

$$
m_{n r s i}=\int_{0}^{e_{n}} a^{r} d a \int_{0}^{e_{n}-a} b^{s} e^{-c_{1}} q_{i}(a, b) d b .
$$

In Appendix A, we showed that

$$
m_{n r s i} \approx m_{r s i}=\int_{0}^{\infty} a^{r} d a \int_{0}^{\infty} e^{-c_{1}} q_{i}(a, b) d b=\mathcal{F}_{r s} q_{i}(a, b) .
$$

Now apply Theorem 2.1.

\section{The joint distribution and moments of $\mathrm{Z}_{n}$}

The following corollary follows easily from Theorems 2.1 and 2.2. They use the notation $\mathbf{Z}_{n}$ of (9) and $a_{r}, \bar{a}_{r}, b_{r}, \bar{b}_{r}$ of (10). Set $\mathbf{z}=\left(z_{1}, z_{2}\right) \in R^{2}, a=z_{1} / A_{1}$ and $b=z_{2} / B_{1}$. Then

$$
\begin{aligned}
& c_{r}=z_{1}^{r} \bar{a}_{r}+z_{2}^{r} \bar{b}_{r}, a_{30}=C_{3}(\overline{\mathbf{a}})=a_{3}+\bar{a}_{2}+1 / 3, a_{21}=\bar{a}_{2}+1, \\
& a_{40}=\left(\bar{a}_{2}+1 / 2\right)^{2} / 2=a_{2}^{2} / 2+\bar{a}_{2} / 2+1 / 8, a_{22}=-a_{2} b_{2}+\bar{a}_{2} / 2+\bar{b}_{2} / 2+3 / 4 .
\end{aligned}
$$

Corollary 3.1 gives expansions for the joint distribution function of $\mathbf{Z}_{n}$, the joint density function of $\mathbf{Z}_{n}$ and the product moments of $\mathbf{Z}_{n}$. 
Corollary 3.1 For $d_{i}$ of (21) and $Q_{i}$ of (17),

$$
\text { Prob. }\left(\mathbf{Z}_{n}>\mathbf{z}\right)=\operatorname{Prob} .\left(Z_{n 1}>z_{1}, Z_{n 2} \geq z_{2}\right)=e^{-z_{1}-z_{2}} \sum_{i=0}^{\infty} Q_{i} n^{-i} \text {. }
$$

For $A_{1}, B_{1}$ of (7), $Q_{i}$ of (17) and $p_{n}(a, b), q_{i}(a, b)$ of Theorem 2.1, $\mathbf{Z}_{n}$ has joint density function

$$
p_{n}^{\prime}(\mathbf{z})=p_{n}(a, b) / A_{1} B_{1}=e^{-z_{1}-z_{2}} \sum_{i=0}^{\infty} q_{i}^{\prime} n^{-i},
$$

where $q_{i}^{\prime}=q_{i}(a, b) / A_{1} B_{1}=J^{\prime} Q_{i}, J^{\prime}=J_{1}^{\prime} J_{2}^{\prime}$ and $J_{i}^{\prime}=\partial / \partial z_{i}$. For $A_{1}, B_{1}$ of (7) and $m_{r s i}$ of Theorem 2.2,

$$
E Z_{n 1}^{r} Z_{n 2}^{s} \approx \sum_{i=0}^{\infty} M_{r s i} n^{-i}
$$

where $M_{r s i}=A_{1}^{r} B_{1}^{s} m_{r s i}$.

Example 3.1 For any continuous distribution function $F(x)$ on $R,\left(F\left(X_{1}\right), F\left(m_{n}\right), F\left(M_{n}\right)\right)$ has the same distribution as $\left(X_{1}, m_{n}, M_{n}\right)$ from $U[0,1]$, the uniform distribution on $[0,1]$. So, $\left(n F\left(m_{n}\right), n-n F\left(M_{n}\right)\right)$ has the same distribution as $\left(n m_{n}, n-n M_{n}\right)$ from $U[0,1]$.

\section{Marginal expansions of $\mathbf{Y}_{n}$ for $A_{1}>0, B_{1}>0$}

A dramatic simplification occurs when dealing with marginals. This is because $b=0$ implies

$$
c_{r}=a^{r} A_{r}, \widetilde{B}_{r, k}(\mathbf{c})=a^{r} \widetilde{B}_{r, k}(\mathbf{A}), C_{r}(\mathbf{c})=a^{r} C_{r}(\mathbf{A}), d_{i}=a^{i+1} D_{i}, \widetilde{B}_{i, k}(\mathbf{d})=a^{i+k} \widetilde{B}_{i, k}(\mathbf{D})
$$

for $D_{i}=C_{i+1}(\mathbf{A}), C_{r}(\mathbf{c})$ of Lemma $2.1, \mathbf{c}=\left(c_{1}, c_{2}, \ldots\right), \mathbf{A}=\left(A_{1}, A_{2}, \ldots\right), \mathbf{d}=\left(d_{1}, d_{2}, \ldots\right)$ and $\mathbf{D}=\left(D_{1}, D_{2}, \ldots\right)$. Similarly, $a=0$ implies

$$
c_{r}=b^{r} \bar{B}_{r}, \widetilde{B}_{r, k}(\mathbf{c})=b^{r} \widetilde{B}_{r, k}(\overline{\mathbf{B}}), C_{r}(\mathbf{c})=b^{r} C_{r}(\overline{\mathbf{B}}), d_{i}=b^{i+1} D_{i}, \widetilde{B}_{i, k}(\mathbf{d})=b^{i+k} \widetilde{B}_{i, k}(\mathbf{D})
$$

for $D_{i}=C_{i+1}(\overline{\mathbf{B}}), \mathbf{c}=\left(c_{1}, c_{2}, \ldots\right), \overline{\mathbf{B}}=\left(\bar{b}_{1}, \bar{b}_{2}, \ldots\right), \mathbf{d}=\left(d_{1}, d_{2}, \ldots\right)$ and $\mathbf{D}=\left(D_{1}, D_{2}, \ldots\right)$.

Putting $b=0$ or $a=0$ in Theorem 2.1 gives the marginal distribution functions in terms of $C_{r}(\mathbf{A})$ and $C_{r}(\mathbf{B})$. Corollary 4.1 gives the marginal distribution and density functions of $\mathbf{Y}_{n}$. The marginal moments of $\mathbf{Y}_{n}$ are given in Corollary 4.2.

Corollary 4.1 For $A_{i}, B_{i}$ of (7), $\bar{B}_{i}$ of (13), $D_{i}=C_{i+1}(\mathbf{A}), C_{r}=C_{r}(\mathbf{A})$ of Lemma 2.1 and $Q_{i}$ of Theorem 2.1,

$$
P_{n 1}=\operatorname{Prob} .\left(Y_{n 1}>a\right)=e^{-a A_{1}} \sum_{i=0}^{\infty} Q_{i} n^{-i}
$$

where

$$
Q_{i}=Q_{i}(a, 0)=a^{i} \sum_{k=0}^{i}(-a)^{k} \widetilde{B}_{i, k}(\mathbf{D}) / k !
$$


Similarly,

$$
P_{n 2}=\operatorname{Prob} .\left(Y_{n 2} \geq b\right)=e^{-b B_{1}} \sum_{i=0}^{\infty} Q_{i} n^{-i}
$$

where $Q_{i}=Q_{i}(0, b)$, is given by replacing $a$ and $C_{r}(\mathbf{A})$ above by $b$ and $C_{r}(\overline{\mathbf{B}})$. For $A_{i}$ of $(7), J_{1}$ of (18) and $C_{r}=C_{r}(\mathbf{A}), Y_{n 1}$ of (6) has density function

$$
p_{n 1}(a)=-e^{-a A_{1}} \sum_{i=0}^{\infty} q_{i 1}(a) n^{-i},
$$

where $q_{i 1}(a)=J_{1} Q_{i}(a, 0)$. For $B_{i}$ of (7), $\bar{B}_{i}$ of (13), $J_{2}$ of (18) and $C_{r}=C_{r}(\overline{\mathbf{B}}), Y_{n 2}$ of (6) has density function

$$
p_{n 2}(b)=-e^{-b B_{1}} \sum_{i=0}^{\infty} q_{i 2}(b) n^{-i},
$$

where $q_{i 2}(b)=J_{2} Q_{i}(0, b)$, given by replacing $J_{1} a^{j}$ by $J_{2} b^{j}$ in $q_{i 1}(a)$ above.

Proof: Note that for $J_{i}$ of (18)

$$
J_{1} a^{i}=i a^{i-1}-A_{1} a^{i}, J_{2} b^{i}=i b^{i-1}-B_{1} b^{i} .
$$

Using $P_{n}(a, b)=P_{n}$ and $U_{n}(a, b)$ of $(22)$, we obtain

$$
p_{n 1}(a)=-\partial_{a} P_{n}(a, 0)=-e^{-a A_{1}} J_{1} U_{n}(a, 0),
$$

where

$$
J_{1} U_{n}(a, 0)=\sum_{i=0}^{\infty} q_{i 1}(a) n^{-i}
$$

$q_{i 1}(a)$ is a polynomial of degree $2 i$ in $a$ and $q_{i 2}(b)$ is a polynomial of degree $2 i$ in $b$, say, then

$$
q_{i 1}(a)=\sum_{j=0}^{2 i} q_{i j 1} a^{j}, q_{i 2}(b)=\sum_{j=0}^{2 i} q_{i j 2} b^{j} .
$$

For $q=q(a)$ any function, set

$$
\begin{aligned}
& \mathcal{F}_{1} q=\int_{0}^{\infty} e^{-a A_{1}} q(a) d a, \mathcal{F}_{r 1} q=\mathcal{F}_{1} a^{r} q(a), \\
& \mathcal{F}_{2} q=\int_{0}^{\infty} e^{-b B_{1}} q(b) d b, \mathcal{F}_{r 2} q=\mathcal{F}_{1} b^{r} q(b),
\end{aligned}
$$

when these integrals exist. Set

$$
G_{r}(A)=\int_{0}^{\infty} a^{r} e^{-a A} d a=r ! A^{-r-1}
$$


for $A>0$. For $J_{i}$ of (18), set

$$
\begin{aligned}
& N_{r j}=\mathcal{F}_{r 1} J_{1} a^{j}=\int_{0}^{\infty}\left(J_{1} a^{j}\right) a^{r} e^{-a A_{1}} d a=-r(r+j-1) ! A_{1}^{-r-j}, \\
& M_{s j}=\mathcal{F}_{s 2} J_{2} b^{j}=\int_{0}^{\infty}\left(J_{2} b^{j}\right) e^{-b B_{1}} d b=-s(s+j-1) ! B_{1}^{-s-j} .
\end{aligned}
$$

From (28) and Corollary 4.1, we obtain

Corollary 4.2 For $A_{1}, B_{1}$ of (7), $p_{n 1}, p_{n 2}$ of Corollary 4.1, $q_{i j 1}, q_{i j 2}$ of (28) and $G_{r}$ of (29),

$$
\begin{aligned}
& E Y_{n 1}^{r} \approx \mathcal{F}_{r 1} p_{n 1}=\sum_{i=0}^{\infty} m_{r 0 i} n^{-i} \\
& E Y_{n 2}^{s} \approx \mathcal{F}_{s 2} p_{n 2}=\sum_{i=0}^{\infty} m_{0 s i} n^{-i}
\end{aligned}
$$

for

$$
\begin{aligned}
& m_{r 0 i}=-\sum_{j=0}^{2 i} q_{i j 1} G_{r+j}\left(A_{1}\right), \\
& m_{0 s i}=-\sum_{j=0}^{2 i} q_{i j 2} G_{s+j}\left(B_{1}\right) .
\end{aligned}
$$

\section{$5 \quad$ Marginal expansions of $\mathbf{Z}_{n}$ for $A_{1}>0, B_{1}>0$}

Theorems 2.1 and 3.1 of Withers and Nadarajah (2017) gave expansions for the marginal distribution functions, density functions and moments of $Z_{n 1}$ and $Z_{n 2}$, respectively. Here, we take a somewhat different route giving more explicit results. Note that by (16), for $a_{r}, \bar{b}_{r}$ of (10), $\mathbf{a}=\left(a_{1}, a_{2}, \cdots\right)$ and $\overline{\mathbf{b}}=\left(\bar{b}_{1}, \bar{b}_{2}, \ldots\right)$,

$$
C_{r}(\mathbf{A})=A_{1}^{r} C_{r} \text { for } C_{r}=C_{r}(\mathbf{a})
$$

and

$$
C_{r}(\overline{\mathbf{B}})=B_{1}^{r} C_{r} \text { for } C_{r}=C_{r}(\overline{\mathbf{b}}) .
$$

So,

$$
\begin{aligned}
& E Z_{n 1}^{r}=\sum_{i=0}^{\infty} M_{i r 0} n^{-i} \\
& E Z_{n 2}^{s} \approx \sum_{i=0}^{\infty} M_{i 0 s} n^{-i}
\end{aligned}
$$

where $M_{i r 0} \approx A_{1}^{r} m_{i r 0}$ and $M_{i 0 s}=B_{1}^{s} m_{i 0 s}$.

Putting $a=z_{1} / A_{1}$ and $b=z_{2} / B_{1}$ in Section 4 gives Theorem 5.1. The marginal density functions of $\mathbf{Z}_{n}$ are also given in Theorem 5.1. The marginal variance, the marginal standard deviation and the marginal reciprocal standard deviation of $\mathbf{Z}_{n}$ are given in Corollary 5.1. 
Theorem 5.1 For $a_{r}, \bar{b}_{r}$ of (10), $C_{r}=C_{r}(\mathbf{a})$ of (30) and $Q_{i}$ of Corollary 4.1,

$$
\text { Prob. }\left(Z_{n 1}>z_{1}\right)=e^{-z_{1}} \sum_{i=0}^{\infty} n^{-i} Q_{i} \text {. }
$$

Prob. $\left(Z_{n 2}>z_{2}\right)$ is given by replacing $z_{1}$ by $z_{2}$ and $C_{r}(\mathbf{a})$ by $C_{r}(\overline{\mathbf{b}})$ of (31), say

$$
\text { Prob. }\left(Z_{n 2}>z_{2}\right)=e^{-z_{2}} \sum_{i=0}^{\infty} n^{-i} Q_{i} .
$$

For $a_{r}, \bar{b}_{r}$ of (10), $C_{r}=C_{r}(\mathbf{a})$ of (30) and $Q_{i}$ of Corollary 4.1, $Z_{n 1}$ has density function

$$
p_{n}\left(z_{1}\right)=e^{-z_{1}} \sum_{i=0}^{\infty} q_{i} n^{-i}
$$

where $q_{i}=K_{1} Q_{i}$ and $K_{i}=1-\partial_{z_{i}}$. For $C_{r}=C_{r}(\overline{\mathbf{b}})$ of (31) and $Q_{i}$ of Corollary 4.1, $Z_{n 2}$ has density function $p_{n}\left(z_{2}\right)$ given by replacing $z_{1}$ by $z_{2}$ and $C_{r}(\mathbf{a})$ by $C_{r}(\overline{\mathbf{b}})$.

Corollary 5.1 For $C_{r}$ of (30),

$$
\sigma\left(Z_{n 1}\right)^{2}=\operatorname{var}\left(Z_{n 1}\right) \approx \sum_{i=0}^{\infty} v_{i} n^{-i}
$$

and

$$
\sigma\left(Z_{n 1}\right)^{ \pm 1} \approx \sum_{i=0}^{\infty} \sigma_{i}( \pm) n^{-i}
$$

with $v_{i}$ and $\sigma_{i}( \pm)$ determined by the proof. This also holds with $M_{r 0 i}, Z_{n 1}$ and $C_{r}$ replaced by $M_{0 r i}$, $Z_{n 2}$ and $C_{r}$ of (31).

Proof: We use the convolution notation

$$
\left(\sum_{i=0}^{\infty} f_{i} x^{i}\right)\left(\sum_{i=0}^{\infty} g_{i} x^{i}\right)=\sum_{i=0}^{\infty} e_{i} x^{i},
$$

where

$$
e_{i}=f_{i} \otimes g_{i}=\sum_{j=0}^{i} f_{j} g_{i-j} .
$$

If there is more than one subscript, the convolution is for the last. So, taking $f_{i}=M_{10 i}, v_{i}=$ $M_{20 i}-e_{i}$ and $e_{i}=M_{i 10} \otimes M_{i 10}$ gives

$$
\left[\operatorname{var}\left(Z_{n 1}\right)\right]^{r / 2} \approx \sum_{i=0}^{\infty} \sigma_{i}( \pm) n^{-i}
$$

where

$$
\sigma_{i}( \pm)=\sum_{k=0}^{i}\left(\begin{array}{c}
r / 2 \\
k
\end{array}\right) \widetilde{B}_{i, k}, \widetilde{B}_{i, k}=\widetilde{B}_{i, k}(\mathbf{v})
$$


and $\mathbf{v}=\left(v_{1}, v_{2}, \ldots\right)$. The proof is complete.

The results of Sections 4 and 5 for the marginal distribution functions and moments of $Y_{n 1}$ and $Z_{n 1}$ do not require any conditions on $\left\{B_{i}\right\}$. In fact, they also hold for $F(x)$ on $\left[w_{1}, \infty\right)$. Similarly, the results of Sections 4 and 5 for the marginal distribution functions and moments of $Y_{n 2}$ and $Z_{n 2}$ do not require any conditions on $\left\{A_{i}\right\}$; they also hold for $F(x)$ on $\left(-\infty, w_{2}\right]$.

\section{Correlation and extensions}

Corollary 6.1 gives expansions for the covariance and correlation between $Z_{n 1}$ and $Z_{n 2}$.

Corollary 6.1 We have

$$
\operatorname{cov}\left(Z_{n 1}, Z_{n 2}\right) \approx \sum_{i=0}^{\infty} k_{i} n^{-i}
$$

and

$$
\operatorname{corr}\left(Z_{n 1}, Z_{n 2}\right) \approx \sum_{i=0}^{\infty} r_{i} n^{-i}
$$

with $k_{i}$ and $r_{i}$ determined by the proof.

Proof: Apply Theorem 5.1. Set $k_{i}=M_{11 i}-M_{10 i} \otimes M_{i 01 i}$ and $\sigma_{i}[a]=\sigma_{i}(-1)$ of Corollary 5.1. Then

$$
\sum_{i=0}^{\infty} r_{i} n^{-i}=\left(\sum_{i=0}^{\infty} k_{i} n^{-i}\right)\left(\sum_{i=0}^{\infty} \sigma_{i}[a] n^{-i}\right)\left(\sum_{i=0}^{\infty} \sigma_{i}[b] n^{-i}\right)
$$

for $r_{i}=k_{i} \otimes \sigma_{i}[a] \otimes \sigma_{i}[b]$.

Withers and Nadarajah (2017) also dealt with the case, where $w_{1}=0$ and for some $g, F(x) / x^{g}$ has finite derivatives at zero.

Finally, we consider the case $A_{1}=0$ or $B_{1}=0$. Let $I, J$ be the lowest integers such that $A_{I} \neq 0, B_{J} \neq 0$. Then one can get similar expansions for $\mathbf{Z}_{n}$, where $Z_{n 1}=n\left(m_{n}-w_{1}\right)\left(n /\left|A_{I}\right|\right)^{1 / I}$ and $Z_{n 2}=n\left(w_{2}-M_{n}\right)\left(n /\left|B_{J}\right|\right)^{1 / J}$. See also Theorems 2.2 and 3.2 in Withers and Nadarajah (2017).

\section{Appendix A}

Here, we give an exact formula for $m_{n r s 0}$ of (27). The method extends to general $m_{n r s i}$.

Theorem A.1 Set $v_{n}=e_{n}\left(A_{1}-B_{1}\right)$ for $e_{n}$ of (8) and $A_{1}, B_{1}$ of (7). If $A_{1}>B_{1}$, then for $m_{n r s 0}$ of (27)

$$
m_{n r s 0} A_{1}^{r} B_{1}^{s}=r ! s !-s ! e^{-A_{1} e_{n}} \sum_{j=0}^{r}(r)_{j}\left(A_{1} e_{n}\right)^{r-j}-\sum_{k=0}^{s}(s)_{k} L_{n, r, s-k}
$$


for

$$
\begin{aligned}
& L_{n r s}=e^{-B_{1} e_{n}}\left(1-B_{1} / a_{1}\right)^{-r-1} I_{n r s}, I_{n r s}=S_{n r s}-e^{-v_{n}} T_{n r s}, \\
& S_{n r s}=\sum_{i=0}^{s}\left(\begin{array}{l}
s \\
i
\end{array}\right)\left(B_{1} e_{n}\right)^{s-i}\left[-B_{1} /\left(A_{1}-B_{1}\right)\right]^{i}(r+i) !, \\
& T_{n r s}=\sum_{i=0}^{s}\left(\begin{array}{l}
s \\
i
\end{array}\right)\left(B_{1} e_{n}\right)^{s-i}\left[-B_{1} /\left(A_{1}-B_{1}\right)\right]^{i} \sum_{k=0}^{r+i}(r+i)_{k} v_{n}^{k}=\left(B_{1} e_{n}\right)^{s} v_{n}^{r} T_{r s}, \\
& T_{r s}=\sum_{i=0}^{s}\left(\begin{array}{l}
s \\
i
\end{array}\right)(r+i) !(-1)^{i} .
\end{aligned}
$$

If $A_{1}<B_{1}$, swap $A_{1}, B_{1}, r, s$ with $B_{1}, A_{1}, s$, r. If $A_{1}=B_{1}$, then

$$
M_{n r s 0}=r ! s !-r ! e^{-A_{1} e_{n}} K_{n r s}
$$

for

$$
\begin{aligned}
& K_{n r s}=s ! \sum_{j=0}^{r}(r)_{j}\left(A_{1} e_{n}\right)^{r-j}+\sum_{k=0}^{s}(s)_{k} I_{n r, s-k}, \\
& I_{n r s}=\left(A_{1} e_{n}\right)^{r+s+1} I_{r s}, I_{r s}=\sum_{i=0}^{s}\left(\begin{array}{c}
s \\
i
\end{array}\right)(-1)^{i} /(r+i+1) .
\end{aligned}
$$

Furthermore, $M_{n r s 0} \approx M_{r s 0}=r ! s !$ since

$$
M_{n r s 0}=r ! s !-r ! e^{-B_{1} e_{n}}\left(B_{1} e_{n}\right)^{s}\left[1+O\left(n^{-1}\right)\right]
$$

if $A_{1}>B_{1}$ and

$$
M_{n r s 0}=r ! s !-e^{-A_{1} e_{n}}\left(A_{1} e_{n}\right)^{r+s+1} I_{r s}\left[1+O\left(n^{-1}\right)\right]
$$

if $A_{1}=B_{1}$.

Proof: Note that

$$
\begin{aligned}
M_{n r s 0} & =m_{n r s 0} / A_{1} B_{1}=\int_{0}^{e_{n}} a^{r} e^{-a A_{1}} d a \int_{0}^{e_{n}-a} b^{s} e^{-b B_{1}} d b \\
& =A_{1}^{-r-1} B_{1}^{-s-1} \int_{0}^{A_{1} e_{n}} u^{r} e^{-u} d u \int_{0}^{y_{n}(u)} t^{s} e^{-t} d t
\end{aligned}
$$

for $y_{n}(u)=\left(e_{n}-u / A_{1}\right) B_{1}$. By equations (6.5.3) and (6.5.32) in Abramowitz and Stegun (1968),

$$
\int_{x}^{\infty} t^{g-1} e^{-t} d t=x^{g-1} e^{-x} \sum_{k=0}^{\infty}(g-1)_{k} x^{-k} .
$$

This expansion diverges unless $g=1,2, \ldots$; in that case we can replace

$$
\sum_{k=0}^{\infty}
$$


in (32) by

$$
\sum_{k=0}^{g-1}
$$

So, for $y_{n}=y_{n}(u)$,

$$
\int_{0}^{y_{n}} t^{s} e^{-t} d t=s !-e^{-y_{n}} \sum_{k=0}^{s}(s)_{k} y_{n}^{s-k}
$$

implies

$$
L_{n r s}=\int_{0}^{A_{1} e_{n}} u^{r} e^{-u-y_{n}} y_{n}^{s} d u
$$

and

$$
I_{n r s}=\int_{0}^{\alpha A_{1} e_{n}} u^{r} e^{-\alpha u} y_{n}^{s} d u
$$

at $\alpha=1-B_{1} / A_{1}$. In the case $A_{1}>B_{1}$,

$$
\begin{aligned}
I_{n r s} & =\sum_{i=0}^{s}\left(\begin{array}{l}
s \\
i
\end{array}\right)\left(B_{1} e_{n}\right)^{s-i}\left[-B_{1} /\left(A_{1}-B_{1}\right)\right]^{i} K_{n, r+i}, \\
K_{n r} & =\int_{0}^{v_{n}} x^{r} e^{-x} d x=r !-e^{-v_{n}} \sum_{k=0}^{r}(r)_{k} v_{n}^{k}=r !+O\left(e^{-v_{n}} v_{n}^{r}\right) .
\end{aligned}
$$

In the case $A_{1}=B_{1}$,

$$
I_{n r s}=\int_{0}^{A_{1} e_{n}} u^{r}\left(A_{1} e_{n}-u\right)^{s} d u=\sum_{i=0}^{s}\left(\begin{array}{l}
s \\
i
\end{array}\right)\left(A_{1} e_{n}\right)^{s-i}(-1) \int_{0}^{A_{1} e_{n}} u^{r+i} d u .
$$

The proof is complete.

This illustrates how replacing $e_{n}$ by $\infty$ in the upper integral limits of (26) introduces errors that are exponentially small, that is, $m_{n r s i} \approx m_{r s i}$.

\section{Acknowledgments}

The authors would like to thank the Editor and the two referees for careful reading and comments which improved the paper.

\section{References}

[1] Abramowitz, M. and Stegun, I. A., eds. (1968). Handbook of Mathematical Functions with Formulas, Graphs, and Mathematical Tables. Applied Mathematics Series 55, Washington DC. 
[2] Choi, B. S. and Roh, J. H. (2013). On the trivariate joint distribution of Brownian motion and its maximum and minimum. Statistics and Probability Letters, 83, 1046-1053.

[3] Comtet, L. (1974). Advanced Combinatorics: The Art of Finite and Infinite Expansions. Springer Verlag, New York.

[4] Leadbetter, M. R., Lindgren, G. and Rootzen, H. (1983). Extremes and Related Properties of Random Sequences and Processes. Springer Verlag, New York.

[5] Liao, X. and Peng, Z. (2015). Asymptotics for the maxima and minima of Husler-Reiss bivariate Gaussian arrays. Extremes, 18, 1-14.

[6] Lu, Y. and Peng, Z. (2017). Maxima and minima of independent and non-identically distributed bivariate Gaussian triangular arrays. Extremes, 20, 187-198.

[7] Shiryaeva, L. K. (2013). The joint distribution of the standardized maximum and standardized minimum for a normal sample.

[8] Withers, C. S. and Nadarajah, S. (2015). The joint distribution of the maximum and minimum of an $\operatorname{AR}(1)$ process. Statistics and Probability Letters, 99, 77-84.

[9] Withers, C. S. and Nadarajah, S. (2017). The distribution of the minimum of a positive sample. Unpublished Technical Report, School of Mathematics, University of Manchester, UK. 